\title{
Development of micro-Laue technique at Shanghai Synchrotron Radiation Facility for materials sciences
}

\author{
Chenyu Ren ${ }^{1 \dagger}$, Li Jiang ${ }^{2 \dagger}$, Jiawei Kou ${ }^{1 \dagger}$, Shuai $\mathrm{Yan}^{3}, \mathrm{Li} \mathrm{Li}^{3}$, Mengting Liu ${ }^{3}$, Xiaohao Dong ${ }^{3}$, \\ Kai Chen ${ }^{1^{*}}$, Zhongliang $\mathrm{Li}^{3^{*}}$, Zhijun $\mathrm{Li}^{2^{*}}$, Xiaoxu Huang ${ }^{4}$ and Renzhong Tai ${ }^{3^{*}}$
}

\begin{abstract}
Synchrotron radiation-based micro-Laue technique has showcased great application potentials in materials science study for its unprecedented crystal orientation and lattice strain/stress resolution. Here we report the updated progress in the development of the micro-Laue technique on the $\mathrm{X}$-ray test beamline at Shanghai Synchrotron Radiation Facility. So far, $40 \mu \mathrm{m}(\mathrm{h}) \times 50 \mu \mathrm{m}(\mathrm{v}) \mathrm{X}$-ray beam spot is routinely obtained, with the convergent angle of $0.2 \mathrm{mrad}(\mathrm{h})$ $\times 0.12 \mathrm{mrad}(\mathrm{v})$. Area scans are conducted on a GH3535 Nibased superalloy base metal and weld joint with the same chemical composition. By analyzing the tremendous amount of Laue diffraction patterns using in-house developed software packages, the crystal orientation, elastic strain, and defect distributions are mapped and investigated. Such a successful proof-of-principle study offers first-hand experience on the further optimization of the design and construction of the scanning micro-Laue facility on the superbend beamline with improved spatial resolution and multiple functions for simultaneous chemical fluorescence mapping and in-situ microstructural evolution studies. The micro-Laue diffraction beamline at Shanghai Synchrotron Radiation Facility will provide a versatile and powerful tool for the orientation and strain/stress mapping combined with phase identification with micron-sized spatial resolution.
\end{abstract}

Keywords: synchrotron light source, micro-Laue diffraction, orientation/strain mapping, peak profile analysis, Ni-based superalloy

\section{INTRODUCTION}

According to the classic definition of Laue diffraction, the incident X-ray has to be continuous spectra and the sample must be a single crystal [1]. Synchrotron radiation sources, originally parasitizing on electron accelerator and energy storage ring used for high-energy physics research, have provided huge promotion to academic and industrial research, and updates and expansion of scientific and technological concepts. The synchrotron scanning X-ray micro-Laue diffraction technique takes advantage of the micro- or even nano-sized high-flux polychromatic X-ray beam produced from synchrotron radiation light source with the assistant of focusing optics. Equipped with high-accuracy scanning stage and largearea two-dimensional (2D) detectors, Laue diffraction patterns can be obtained on any crystalline metals, materials and minerals with grain sizes bigger than the X-ray beam size in single point, line-scan, and surface-area-scan modes [2]. Further development of the differential aperture in the manner of wire scan has offered the resolution in the depth dimension and realized the so-called 3D micro-Laue diffraction [3]. By indexing the Laue diffraction patterns recorded at each scanning position, the local crystalline information is extracted, including the phase, orientation, elastic and plastic strain/stress tensor components, and dislocation content.

Compared with charged particle-based material characterization techniques such as electron and focused ion beam microscopy, micro-Laue diffraction offers some

\footnotetext{
${ }^{1}$ Center for Advancing Materials Performance from the Nanoscale (CAMP-Nano), State Key Laboratory for Mechanical Behavior of Materials, Xi'an Jiaotong University, Xi'an 710049, China

${ }^{2}$ Center for Thorium Molten Salt Reactor System, Shanghai Institute of Applied Physics, Chinese Academy of Sciences, Shanghai 201800, China

${ }^{3}$ Shanghai Synchrotron Radiation Facility, Shanghai Advanced Research Institute, Chinese Academy of Sciences, Shanghai 201204, China

${ }^{4}$ International Joint Laboratory for Light Alloys (MOE), College of Materials Science and Engineering, Chongqing University, Chongqing 400044, China

$\uparrow$ These authors contributed equally to this work.

* Corresponding authors (emails: kchenlbl@gmail.com (Chen K); lizhongliang@zjlab.org.cn (Li Z); lizhijun@sinap.ac.cn (Li Z); tairenzhong@zjlab.org.cn (Tai R))
} 
important advantages in terms of greater penetration capability so that bulky and buried materials at the depths of several to several hundred microns are probed, and thus no delicate surface polishing, as electron backscatter diffraction (EBSD) requires, and/or thin sectioning, as transmission electron microscopy (TEM) demands, is necessary. Therefore, the artifacts that are frequently introduced during sample preparation procedures are greatly mitigated. The micro-Laue diffraction technique measures the lattice strain and crystallographic orientation with unprecedented accuracy compared with any other microscopic methods. Moreover, combining fine probe size and scanning capability, micro-Laue diffraction provides decent spatial resolution (hundreds of $\mathrm{nm}$ to $\mu \mathrm{m})$ and sampling area/volume (mm size) simultaneously, which bridges the atomic and macroscopic scales and showcases applications in the research fields of materials science, physics, chemistry, electronics, and earth and environmental sciences. Taking advantage of $3 \mathrm{D}$ microdiffraction, strain/stress concentration and deformation-induced cracking mechanism in bulk austenite steel are unraveled at the submicron scale [4]. The magnitude and direction of the highly non-uniform residual strain distribution in additively manufactured (3D printed) superalloy single crystals are mapped, enabling the design of strain releasing heat treatment protocol [5]. For novel functional materials, the electric conductivity behavior depending on domain structures, as well as crystal structure and phase transition dependence of temperature, electric current, and chemical doping are comprehensively studied with the assistance of in situ testing stages [6-9]. For even more complicated systems such as natural minerals and biominerals, micro-Laue diffraction also finds its unique applications, to uncover the deformation history as a potential palaeo-piezometer [10] and the microstructure-toughness relation [11]. Different from sharp Laue peaks obtained from defect free crystals, the streaking of Laue diffractions is observed from a specimen in which geometrically necessary dislocations (GNDs), constituting arrays of dislocations of the same sign, accommodate local crystal lattice bending. GNDs can be measured with both EBSD and micro-Laue, while the latter offers much improved accuracy because of better orientation resolution [12]. Statistically stored dislocations (SSDs) in contrast produce an angularly isotropic broadening of Laue reflections [13], which cannot be detected using EBSD. The application of dislocation study using TEM is limited to low dislocation densities (not exceeding $10^{14} \mathrm{~m}^{-2}$ ) and small area in thin foils, while scanning micro-Laue technique breaks through these barriers [14].

Micro-Laue diffraction beamlines have been designed and constructed in several synchrotron radiation light sources. Beamline 34-ID-E at the Advanced Photon Source (APS) in Argonne National Laboratory, USA, is the first micro-Laue beamline on the earth, featured by $0.3 \mu \mathrm{m} \times 0.3 \mu \mathrm{m}$ focused polychromatic beam with an energy range from 7 to $30 \mathrm{keV}$ and the flux of $\sim 10^{11}$ photons $\mathrm{s}^{-1}$ in white beam mode [15]. The high energy and flux ensure the strong signals from depth and thus make the 3D microdiffraction routinely available for users. Beamline 12.3.2 at the Advanced Light Source (ALS) in Lawrence Berkeley National Lab, USA, is the first microdiffraction beamline in the world with a superbend source (X-ray beam generated by a $6 \mathrm{~T}$ superconducting bending magnet) to avoid highly structured incident spectra and thus simplify the crystal structure refinement [16]. The in-house developed software package, XMAS, is a powerful tool to index and analyze the Laue diffraction patterns from almost all crystal structures. Beamline 21A at Taiwan Photon Source (TPS) of China has achieved sub-100 $\mathrm{nm}$ beam size routinely for general users and assembled a scanning electron microscope with the microdiffraction system, which attracts great attention from the microelectronic community [17]. Ultra-high speed detector has been developed and applied on the micoXAS beamline in Swiss Light Source (SLS) to study the 3D printing of Ti alloys [18]. Micro-Laue diffraction experiments have also been carried out at Diamond [19] and Canadian Light Sources [20] as well as the European Synchrotron Radiation Facility [21]. Some of the key specifications of the corresponding beamlines are summarized in Table 1.

Shanghai Synchrotron Radiation Facility (SSRF) is the first third-generation synchrotron light source of China and has a certain degree of foresight in its design, which is equipped with extensive insertion devices such as multipole wigglers and undulators producing X-rays with high brightness [22]. Along with the improvement and supplements, synchrotron polychromatic micro-Laue diffraction is planned to be implanted on a superbend beamline BL03B. As a proof-of-principle study, the micro-Laue experimental setup is firstly assembled and tried out on the X-ray test beamline (BL09B). In this report, the progress of the micro-Laue project at SSRF is introduced. Micro-sized polychromatic X-ray beam is obtained, and surface 2D scans are carried out on solid-solutionstrengthened Ni-based superalloy GH3535. The collected Laue patterns are analyzed using in-house software packages PPCIA (software copyright registration number 
Table 1 A summary of the micro-Laue beamline specifications all over the world

\begin{tabular}{|c|c|c|c|}
\hline Micro-Laue beamline & Energy range $(\mathrm{keV})$ & Beam size $\left(\mu \mathrm{m}^{2}\right)$ & Uniqueness \\
\hline 34-ID-E@APS & $7-30$ & $0.3 \times 0.3$ & 3D diffraction \\
\hline 12.3.2@ALS & $5-22$ & $\sim 1 \times 1$ & Versatile data analysis software \\
\hline 21A@TPS & $5-30$ & $0.1 \times 0.1$ & Ultra-high sub-100 $\mathrm{nm}$ resolution \\
\hline microXAS@SLS & $3-23$ & $\sim 1 \times 1$ & Ultra-fast $20 \mathrm{kHz}$ data collection \\
\hline B16@DLS & $5-25$ & $\sim 50 \times 50$ & \\
\hline BM32@ESRF & $5-25$ & $\sim 0.5 \times 0.5$ & \\
\hline VESPERS@CLS & $6-30$ & $\sim 4 \times 4$ & \\
\hline $\begin{array}{c}\text { Currently on 09B, finally on } \\
\text { 03B@SSRF }\end{array}$ & $4-30$ & $\begin{aligned} 40 & \times 50 \text { on } 09 B \text { (Jun. } 2020) \\
1 & \times 1.5 \text { on } 03 \mathrm{~B} \text { (designed) }\end{aligned}$ & $\begin{array}{l}\text { High-speed data analysis for simulta- } \\
\text { neous microstructure visualization }\end{array}$ \\
\hline
\end{tabular}

2018SR865737) and LDat (software copyright registration number 2018SR865716). Orientation, lattice strain, and peak width maps are plotted using XtalCAMP developed at Xi'an Jiaotong University [23]. The future development prospect is also discussed briefly.

\section{EXPERIMENTAL SECTION}

The layout of the micro-Laue diffraction experimental setup on the X-ray test beamline, BL09B, at SSRF is displayed schematically in Fig. 1. Electrons are accelerated in the linear accelerator and booster and then transported to the storage ring. On BL09B, synchrotron radiation X-rays are generated by a $1.2726 \mathrm{~T}$ bending magnet. The photon energy ranges from 4 to $30 \mathrm{keV}$ with the critical energy at $10.4 \mathrm{keV}$, the photon flux goes up to $3 \times 10^{11}$ photons s$^{-1}$ @ $10 \mathrm{keV}, 300 \mathrm{~mA}$. The unfocused white beam acceptance angle is $2.5 \mathrm{mrad}$ in horizontal (h for short) and $0.3 \mathrm{mrad}$ in vertical ( $\mathrm{v}$ for short) directions, and the source size is $161 \mu \mathrm{m}(\mathrm{h}) \times 51 \mu \mathrm{m}(\mathrm{v})$, in terms of full width at half maximum (FWHM). The acceptance angle can be adjusted by the $1^{\text {st }}$ white beam slit, which is $18.5 \mathrm{~m}$ from the light source. $2.5 \mathrm{~m}$ downstream from the white beam slit, a water-cooled double crystal monochromator is installed, which can be moved in and out of the beam to enable the rapid switch between white and monochromatic modes. $3 \mathrm{~m}$ further downstream a toroidal mirror is employed to focus the X-ray beam in both horizontal and vertical directions. The toroidal mirror is made of a silicon crystal and coated with 50-nm-thick Rh to secure excellent reflectivity of X-rays up to $20 \mathrm{keV}$. The roughness of the toroidal mirror is less than $3 \AA$ (RMS). Normally, the toroidal mirror is operated at the grazing angle of $2.8 \mathrm{mrad}$, and for even higher energy, the grazing angle is further decreased. Under white beam mode, the toroidal mirror is water cooled to reduce thermal deformation caused by the high thermal load of polychromatic synchrotron radiation.

After passing the $2^{\text {nd }}$ slit, the polychromatic X-ray is finally fine-focused using a pair of perpendicularly configured Kirkpatrick-Baez mirrors (KB mirrors), which sit at $43 \mathrm{~m}$ away from the light source, to achieve micronsized probe. The mirror substrates are made of a super polished plane Si single crystals of $320 \mathrm{~mm} \times 12.5 \mathrm{~mm} \times$ $10 \mathrm{~mm}$ and $220 \mathrm{~mm} \times 12.5 \mathrm{~mm} \times 10 \mathrm{~mm}$ in dimension (both are length $\times$ width $\times$ thickness). The Si substrates are coated with 50-nm $\mathrm{Rh}$. The $\mathrm{KB}$ mirrors work in a chamber filled with He gas to avoid contamination. To prevent thermal effects from the incident X-ray beam, the $\mathrm{KB}$ mirrors are connected with the chamber wall using copper braids. With the $\mathrm{KB}$ mirrors, $4-20 \mathrm{keV}$ white beam or monochromatic beam can be obtained, with $40 \mu \mathrm{m}$ beam size (Fig. 2). The focal length is $120 \mathrm{~mm}$ and

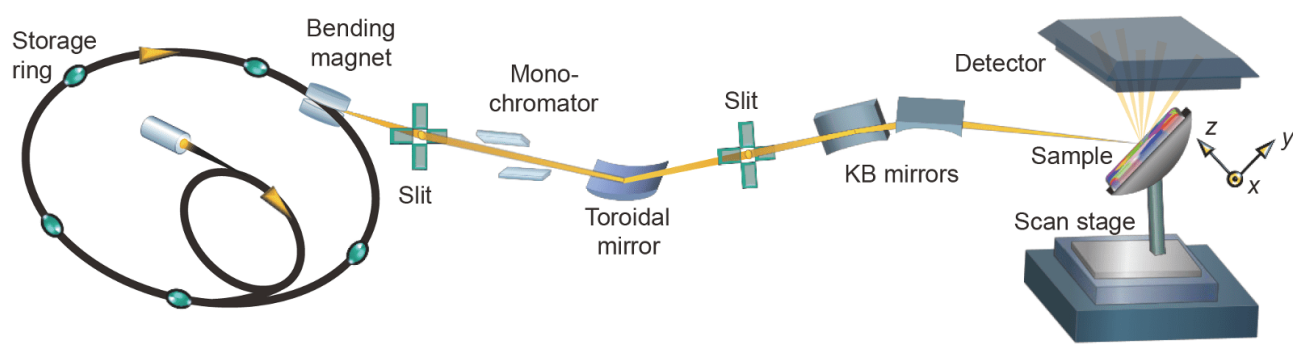

Figure 1 Schematic layout of the micro-Laue diffraction setup on BL09B in SSRF. 

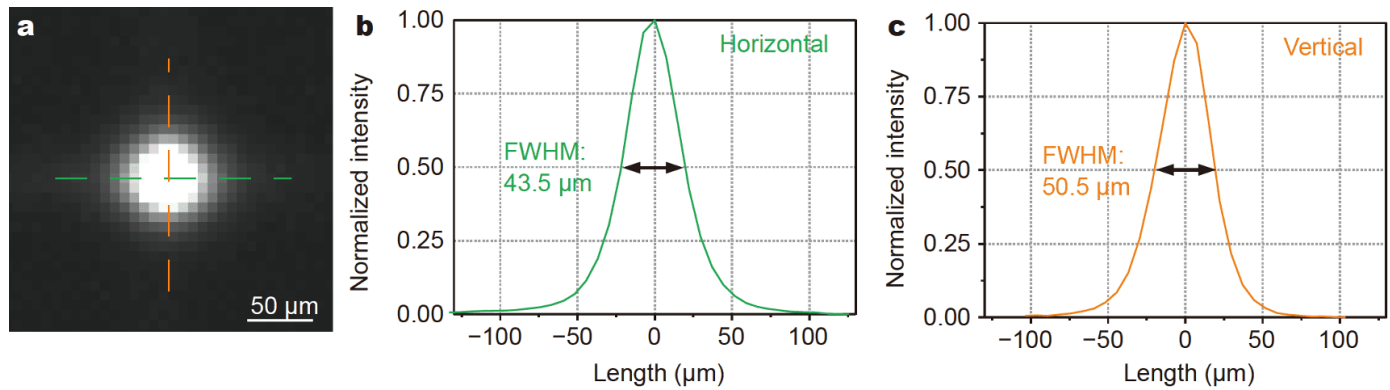

Figure 2 Focused X-ray beam spot shape and size. (a) The X-ray beam spot is symmetric on the scintillator; (b, c) the FWHMs in the horizontal and vertical directions measured to be $\sim 43.5$ and $50.5 \mu \mathrm{m}$, respectively.

the convergence angle is $0.2 \mathrm{mrad}(\mathrm{h}) \times 0.12 \mathrm{mrad}(\mathrm{v})$.

For scanning micro-Laue diffraction study, the samples are mounted firmly on a high-accuracy scan stage with seven motion freedoms, including coarse and fine translational motions in all three dimensions (for simplicity a laboratory coordinate system is established and displayed in Fig. 1) as well as tilting along the $x$-axis to tune the $\mathrm{X}$ ray incident angle. To balance between spatial resolution and detector coverage angle, the sample is usually tilted to $45^{\circ}$ and carefully positioned to have the focal point of the $\mathrm{X}$-ray beam on the sample surface. The polychromatic Laue or monochromatic powder diffraction patterns are collected using a 2D Mercu $1717 \mathrm{~V}$ amorphous Si flat panel detector, which is placed about $417 \mathrm{~mm}$ above the sample surface, at $90^{\circ}$ with respect to the incident X-ray beam. The detector is $3072 \times 3072$ pixels in dimension, and each pixel is $139 \mu \mathrm{m} \times 139 \mu \mathrm{m}$ in size.

\section{DATA ANALYSIS}

On account of enormous synchrotron radiation Laue micro-diffraction patterns collected in each area scan, it is time consuming to index all the Laue patterns one by one sequentially, and it is frequently demanded that the Laue peak profile of each diffraction pattern should be extracted. To fulfill these requirements, custom developed software packages are applied for data mining.

First of all, the experimental parameters need to be input into PPCIA, including the lattice constants of the crystal phases possibly existing in the specimen, energy range of the X-ray, detector dimensions (size and pixel number), and diffraction conditions. The diffraction geometry parameters, including the distance from the sample surface to the active face of the detector, the center channel of the detector, and the relative tilt angle of the detector (yaw and pitch), are dedicatedly refined by taking a Laue diffraction on a strain-free single crystal Si. These parameters are essential to correct Laue pattern indexing, as well as accurate peak profile measurement in reciprocal space. Secondly, the experimental Laue diffraction patterns are loaded into the software and displayed in user-defined false colors (Fig. 3a). Then the background signals mainly come from the fluorescence effects. In PPCIA, the background signals of each Laue pattern are fitted using a 2D generalized Brückner algo-
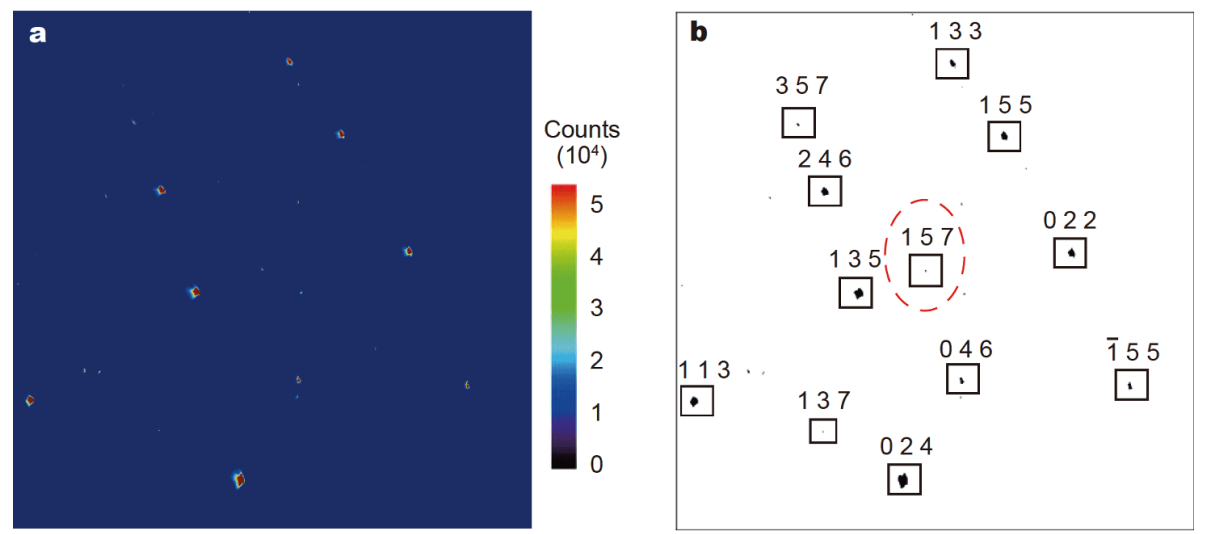

Figure 3 Laue diffraction pattern visualization and indexation using the software package PPCIA. A typical Laue pattern is (a) loaded into the software, (b) binarized, and indexed, with the Miller indices of the Laue reflections marked. 
rithm, which was originally developed to estimate the background for $1 \mathrm{D}$ powder diffraction pattern through a robust smoothing procedure [24]. After the background signals are subtracted from the experimental data, the Laue pattern is binarized and Laue peaks are searched according to a user-defined threshold value, as marked by the black rectangles in Fig. $3 \mathrm{~b}$. It is noted that the black and white colors in Fig. $3 \mathrm{~b}$ are inverted compared with those on the software interface for the sake of display convenience. The position coordinates on the detector $(x$ pixel and $y$-pixel) of each individual Laue diffraction peak are determined by fitting the intensity profile $(I)$ using a 2D Gaussian function. Since it is not an easy task to quantify the intensity of each Laue peak, which is affected by a number of artifacts, especially for polychromatic Xray diffractions, indexing the Laue patterns does not take the intensity information into account. The local crystal orientation is obtained by indexing the Laue pattern, i.e., fitting the position of the Laue diffraction peaks based on the input lattice constants. Once the indexing procedure succeeds, the Miller indices of all indexed Laue peaks show up on the interface, as demonstrated in Fig. 3b. Once indexed, the angles between experimentally recorded diffraction peak positions are compared with the theoretically calculated ones derived from unstrained lattice constants, lattice parameters for a distorted triclinic cell are refined by using least squares fitting, and thus a strain tensor is obtained for each micro-Laue scan position $[25,26]$. The local orientation matrix and strain tensor results are output into .idx and .stn files, respectively, each as a $3 \times 3$ matrix. Taking advantages of the peak position comparison (PPC) algorithm [27], numerous Laue patterns are analyzed automatically, and for each Laue pattern the analyzed results are saved. After all the recorded Laue patterns taken from a microdiffraction scan are analyzed, the spatial distributions of orientation and lattice strain are mapped.

From the fundamental principles of Laue diffraction, the Laue peak width along the long axis, the GND density can be calculated, but the peak width along the short axis is determined by the total dislocation density [28]. If the peak widths along the long- and short-axes are similar, SSDs are dominant in the local diffraction volume. For this reason, the software package LDat is developed for peak profile analysis. An example is given in Fig. 4. The 157 Laue peak from the Ni-based superalloy GH3535 shown in Fig. 3b is enlarged in Fig. 4a. The diffraction peak is firstly converted to the angular $(2 \theta-\chi)$ space (Fig. $4 \mathrm{~b}$ ), and then projected to the $2 \theta$ - and $\chi$-axes, respectively. $2 \theta$, as in most of the diffraction related studies, is defined as the angle between the incident and diffraction beam; $\chi$, following the denotation suggested by Busing \& Levy [29], represents in angle included by two feature planes. One plane is defined by the incident and diffraction X-ray beams, and the other is decided by the incident X-ray beam and the detector center channel. As shown in Fig. 4c, d, respectively, where the peaks are fitted using Gaussian functions, subpeaks are observed, indicating the probable existence of low-angle grain boundaries in the probed sample volume. Gaussian fitting of the major peak in both directions shows significantly different FWHMs, providing apparent evidence to the GNDs in the corresponding subgrain. Users can also use the software to convert the selected Laue peak into the reciprocal $q$-space, which is even more convenient to study the dislocation type and density.

\section{APPLICATIONS}

GH3535 is a solid-solution strengthened Ni-based superalloy designed and fabricated in China, specifically for the future applications of Chinese thorium molten salt reactors. The microstructures of this alloy and its weld joint have been widely investigated using conventional and synchrotron characterization techniques [30]. Here, the synchrotron polychromatic X-ray micro-Laue diffraction was applied to provide additional microstructural information for this alloy, including the crystal orientation, elastic strain, and defect distributions.

\section{Sample preparation}

In this study, the microstructural features of the as-received Ni-based superalloy GH3535 and its weld joint were investigated using synchrotron polychromatic X-ray micro-Laue diffraction. The chemical composition of GH3535 and the corresponding ERNiMo-2 filler material are listed in Table 2. The gas tungsten arc weld joint was prepared on a piece of 20 -mm-thick GH3535 plate using the ERNiMo-2 filler material with a diameter of $1.2 \mathrm{~mm}$. Welding was operated at the voltage of $14 \mathrm{~V}$, current of $200 \mathrm{~A}$, pulse frequency of $2.5 \mathrm{~Hz}$ and $10 \mathrm{~mm} \mathrm{~s}^{-1}$ travel speed under argon shielding gas. Thus the heat input energy was calculated to be $2.8 \mathrm{~kJ} \mathrm{~cm}^{-1}$. More detailed geometric construction and experimental parameters can be found in a previous report [31].

Specimens for the micro-Laue diffraction characterization were cut from the as-received plate (also called base metal in this report) and the fusion zone of the aswelded weld joint. The specimens were mechanically polished using $\mathrm{SiC}$ sandpapers and washed with an ethanol ultrasonic bath for $20 \mathrm{~min}$. Then an area of 

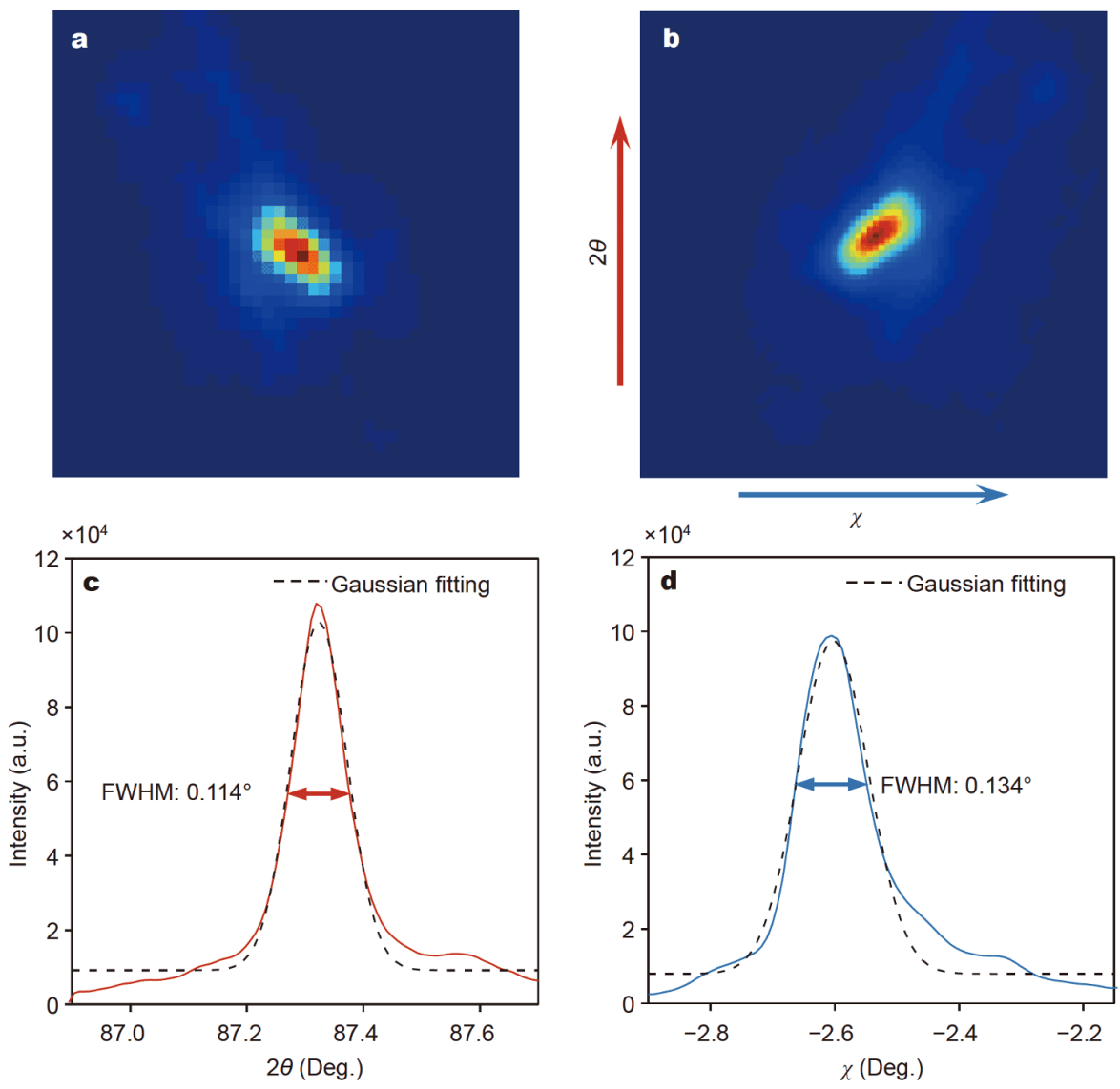

Figure 4 Laue peak profile analysis using the software package LDat. The 157 reflection on the pattern shown in Fig. 3 is (a) enlarged, (b) converted to the $2 \theta-\chi$ space, and (c, d) projected to $2 \theta$ - and $\chi$-axes, respectively.

Table 2 Chemical compositions of the GH3535 plate and ERNiMo-2 welding wire in wt\%

\begin{tabular}{ccccccccccc}
\hline & $\mathrm{Ni}$ & $\mathrm{Cr}$ & $\mathrm{Mo}$ & $\mathrm{Fe}$ & $\mathrm{Si}$ & $\mathrm{C}$ & $\mathrm{Al}$ & $\mathrm{Mn}$ & $\mathrm{S}$ & $\mathrm{P}$ \\
\hline $\begin{array}{c}\text { ERNiMo-2 } \\
\text { filler alloy }\end{array}$ & Bal. & 6.89 & 16.4 & 3.62 & 0.43 & 0.053 & 0.06 & 0.58 & $<0.001$ & 0.002 \\
$\begin{array}{c}\text { GH3535 base } \\
\text { metal }\end{array}$ & Bal. & 6.96 & 16.3 & 3.88 & 0.33 & 0.061 & 0.08 & 0.52 & 0.001 & 0.003 \\
\hline
\end{tabular}

$1050 \mu \mathrm{m} \times 330 \mu \mathrm{m}$ was scanned using the micro-focused $\mathrm{X}$-ray beam on the base metal, denoted as Scan 1, and Scan 2 was performed in the fusion zone for an area of $1740 \mu \mathrm{m} \times 1080 \mu \mathrm{m}$, both with the scanning step size of $30 \mu \mathrm{m}$.

\section{Microstructure of the base metal}

From the orientation map in Fig. 5a, Scan 1 was carried out in a crystal grain with the [011] crystallographic direction parallel to the $x$-scan stage. The filtered intensity map (Fig. 5b) demonstrates more microstructural features [32] and evidences heterogeneous defect distributions in the scanned area. The brighter parts in the filtered intensity map represent sharper diffraction peaks and thus lower defect density, while the darker spots correspond to higher defect density. By calculating the average orientation deviation between each individual scan pixel and its eight closest neighbors, the kernel average misorientation (KAM) map, in the dimension of a degree per unit length, is created and plotted in Fig. 5c. Comparing Fig. 5b and c, similar inclined stripe-shaped features are observed, indicated by the dashed yellow lines on both left and right sides. The misorientation distribution is represented using another way by computing the misorientation angle between the orientation at each scanning position and the one at the bottom left corner 


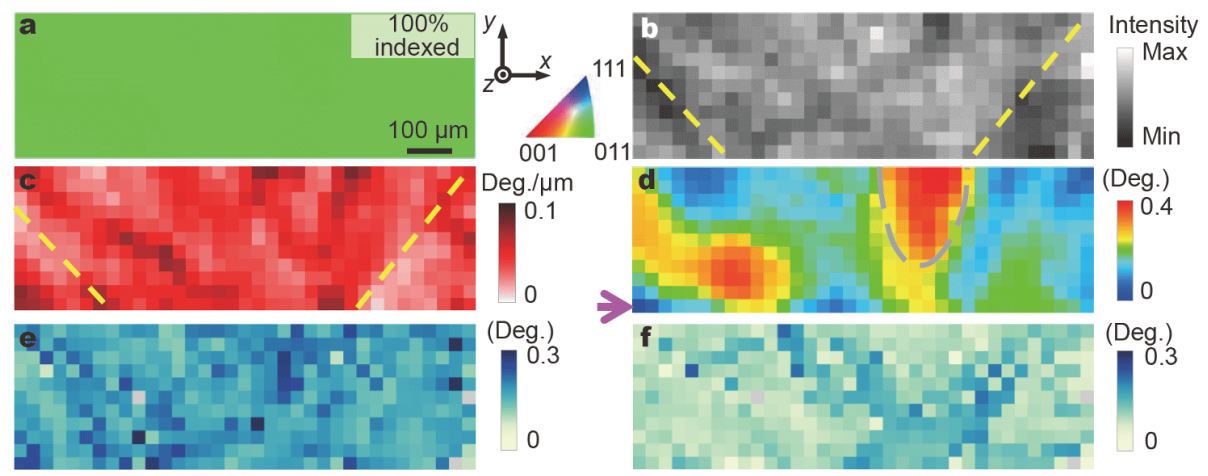

Figure 5 The microstructure distribution of the scanned base metal. (a) Inverse pole figure shows the scanned area contains only one crystal grain. (b) The filtered intensity, (c) KAM, and (d) misorientation of each scanning pixel with respect to the bottom left point show inhomogeneous orientation. (e, f) Non-uniform FWHM of the 135 reflection demonstrates heterogeneous defect distribution.

pointed by the purple arrow, as displayed in Fig. $5 \mathrm{~d}$. The features in this map are closely related to the Laue diffraction peak width, which are demonstrated in Fig. 5e, $\mathrm{f}$ in both $2 \theta$ and $\chi$ directions. Here the 135 Laue peak is picked as an example. Note that the peak widths in both axes are within $0.3^{\circ}$, and it is more broadened in $2 \theta$ axis than in $\chi$ direction.

The diagonal components of the deviatoric strain tensor $\left(\varepsilon_{x x}, \varepsilon_{y y}\right.$, and $\left.\varepsilon_{z z}\right)$ distributions are mapped and shown in Fig. 6. Red and blue pixels stand for tension and compression, respectively, and the color range is from $-1.5 \times 10^{-3}$ to $1.5 \times 10^{-3}$. In general, the scanned volume of the specimen is under tensile strain in the $x$-axis while compressive in the $y$-axis. The out-of-plane strain, $\varepsilon_{z z}$, is significantly released, which is similar to the phenomena previously observed in metallic and mineral materials $[33,34]$. The features observed in the misorientation map can also be visualized in the strain distributions, especially in the $\varepsilon_{z z}$ map, as indicated by the dashed semi-elliptical curve in the strain map as well as Fig. $5 \mathrm{~d}$.

\section{Microstructure of the fusion zone}

Columnar grain structures are observed in the fusion zone, evidenced from both the inverse pole figure (Fig. 7a) and the filtered intensity (Fig. 7b) map. In the inverse pole figure orientation map, especially on the right hand side, quite a portion of Laue patterns are failed to be indexed, due to the high defect density and thus low diffraction peak intensity. The columnar grains are parallel to one another because they grow approximately along the directional temperature gradient caused by the heat transfer from weld pool to base [35,36]. The KAM map shows much higher misorientation in the scanned area in the fusion zone (Fig. 7c), and the misorientation in individual grains, as displayed in Fig. $7 \mathrm{~d}$, is also high
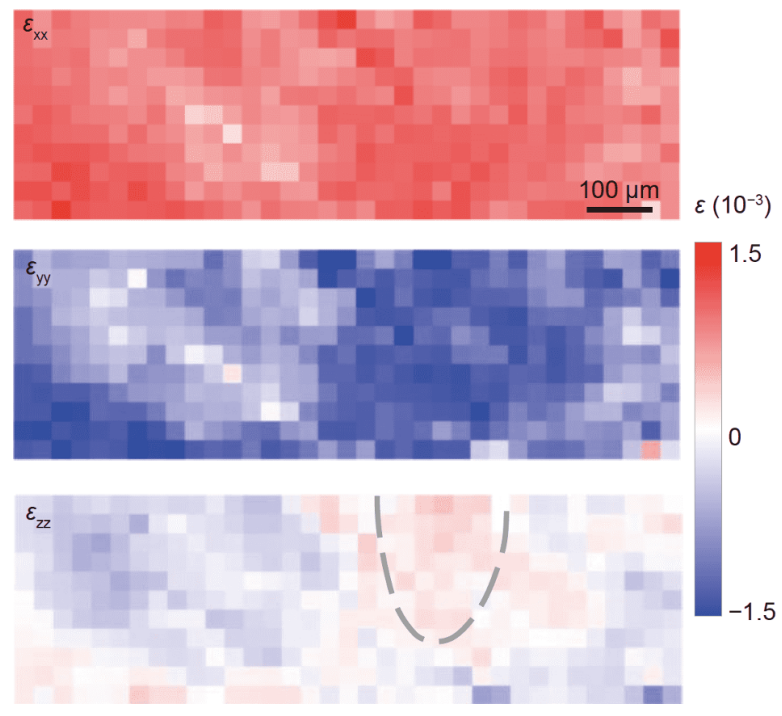

Figure 6 Diagonal strain tensor components of the scanned area in the base metal.

and non-uniform.

For such polycrystalline specimens, it is not straightforward to quantify the diffraction peak width. For an easier comparison, two grains (marked as 1 and 2 in Fig. 7a) are selected for in-depth study. The 135 Laue reflections, with the same Miller indices as those demonstrated in Fig. 5e, f, of both grains are converted and projected onto the $2 \theta$ - and $\chi$-axes. The average peak widths in Grains 1 and 2 spread $0.50^{\circ}$ and $0.47^{\circ}$ along the $2 \theta$-direction, respectively, which are $\sim 2.5$ times as wide as the average peak width in Scan $1\left(\sim 0.18^{\circ}\right)$. In the $\chi$-axis, the Laue reflections measure about $0.29^{\circ}$ in both grains in the fusion zone, which is approximately 1.8 times as broad as the ones in the base metal $\left(0.16^{\circ}\right)$. As a result, the defect density in Scan 2 is much higher than that in Scan 1. 

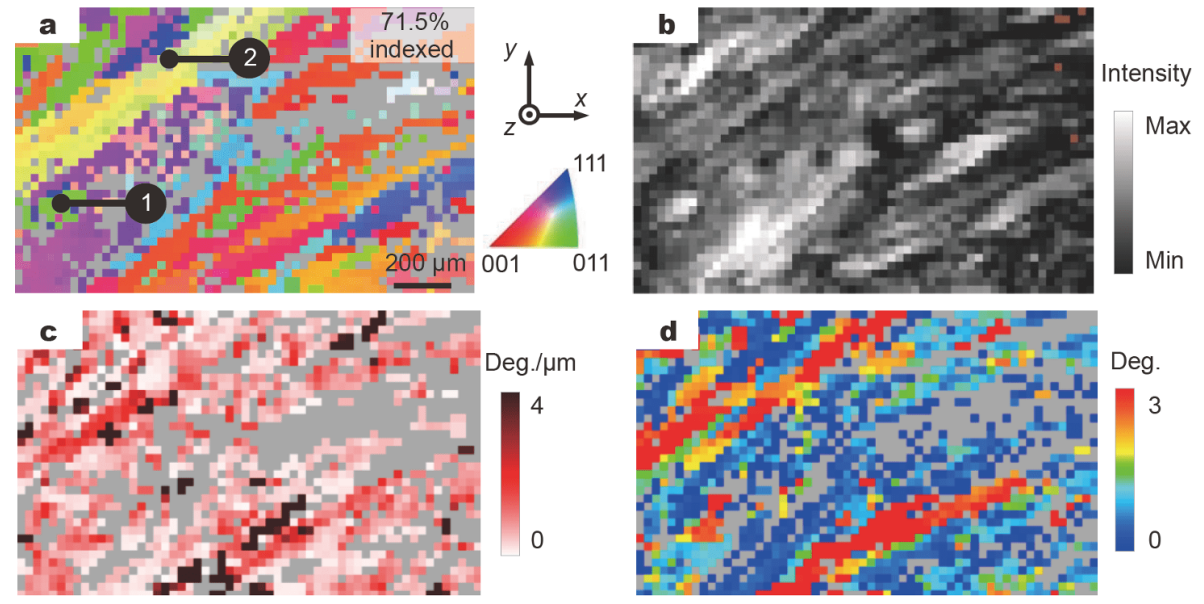

Figure 7 The microstructure distribution of the scanned weld joint area. (a) Inverse pole figure and (b) the filtered intensity show the columnar grain structure; (c) KAM and (d) intragranular misorientation distribution suggest higher orientation gradient than in the base metal.

In the strain tensor component maps in Fig. 8, it is clear that the strains in the fusion zone are by far higher than those in the base metal. The direction and magnitude are more scattered. We believe these are rooted from several sources. First of all, a higher density of microstructural defects, which are distributed more inhomogeneously, exists in this area because of the high cooling rate and crystal growth rate. Secondly, the mismatch of coefficient of thermal expansion and elastic modulus between adjacent crystal grains further contribute to the high magnitude and non-uniformity of the strains. Finally, higher measurement errors are inevitable due to the irregular profile of the Laue diffraction peaks recorded in this area. For a direct comparison, typical Laue patterns from both scanned areas are illustrated in Fig. 9a, b, respectively. The peaks from Scan 1 are sharp, while those from Scan 2 are smeared and splitting. Consequently, the von Mises strains in Scan 1 distribute in a narrow range from $0.5 \times$ $10^{-3}$ to $3 \times 10^{-3}$. However, in Scan 2, the von Mises strains spread from $1 \times 10^{-3}$ to $10 \times 10^{-3}$.

\section{CONCLUSION AND OUTLOOK}

In this study, we report the first realization of synchrotron scanning polychromatic X-ray micro-Laue diffraction technique at SSRF on the test beamline BL09B. With the delicately tuned slits and K-B mirrors, the X-ray beam generated from the bending magnet source is focused down to $40 \mu \mathrm{m} \times 50 \mu \mathrm{m}$. With the aid of scanning stage and $2 \mathrm{D}$ detector, micro-Laue scans are made on two different GH3535 superalloy prototype materials, and thousands of Laue patterns are collected. Using in-house developed data analysis software packages, the micro-

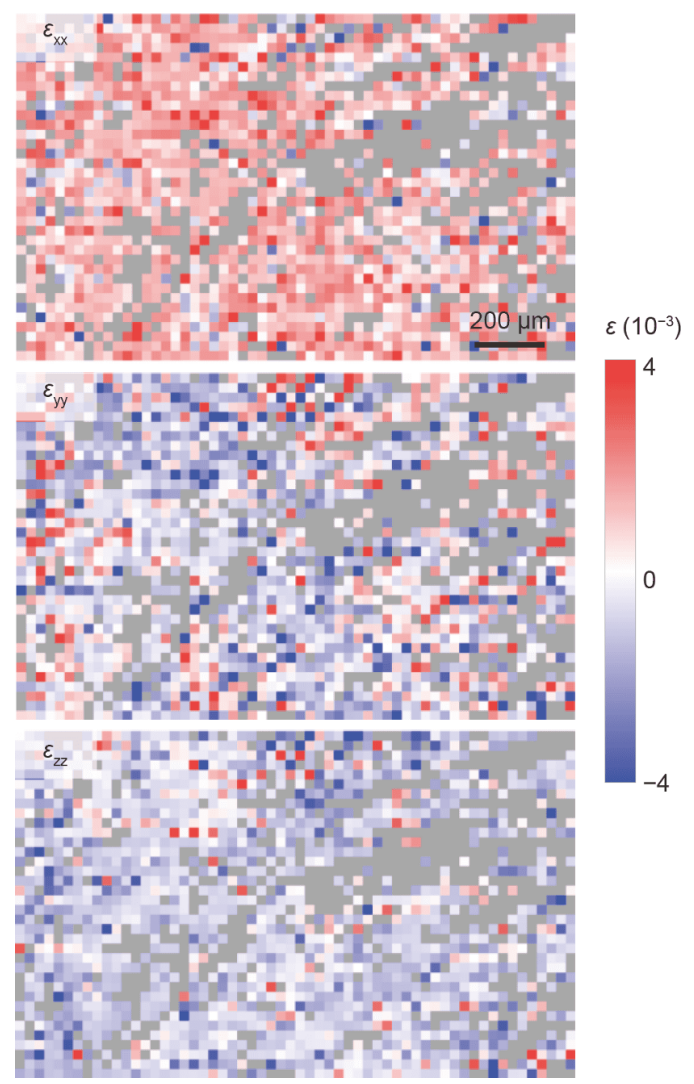

Figure 8 Diagonal strain tensor components of the scanned area in the fusion zone.

structural features, including crystal orientation, orientation gradient, elastic strain tensor, and peak profile, are obtained and visualized with micro-sized spatial resolution. 

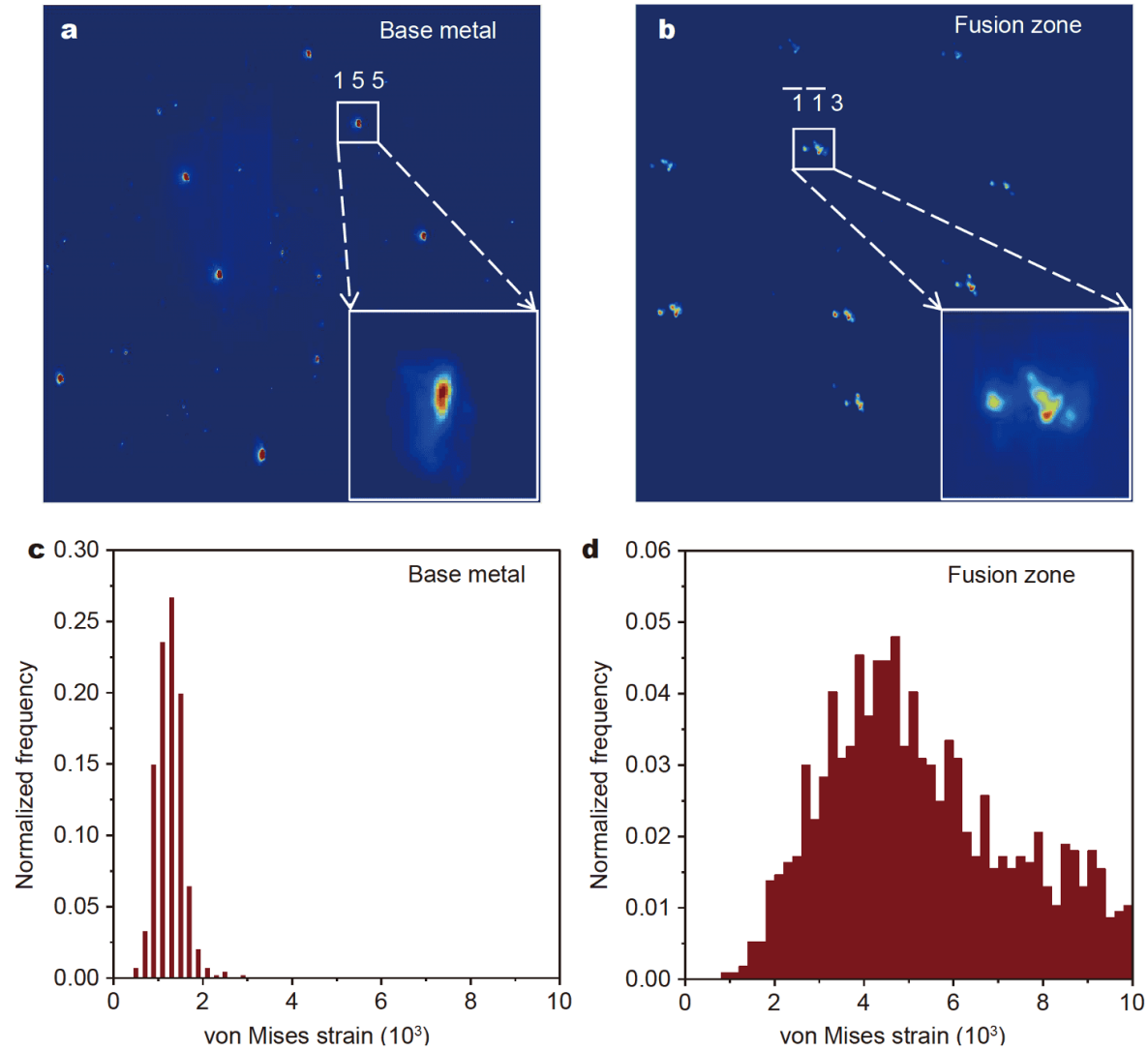

Figure 9 Comparisons of the Laue diffraction peak profiles and strains in both scans. (a,b) The Laue reflections taken on the base metal are sharper than those on the fusion zone; $(c, d)$ the von Mises strains in the base metal are lower and more narrowly distributed than those in the fusion zone.

This study sets an important milestone for the development of the micro-Laue technique at SSRF and paves the way to establish a world-wide leading scientific user facility. Efforts are being made to promote the performance and parameters in the following scopes. First of all, with the superbend source on BL03B, more carefully optimized K-B mirrors and slits, and specially designed optical table with better stability, the beam size can be brought down to micron or even submicron scale to improve the spatial resolution. With the high-flux of the polychromatic X-ray beam and high-speed 2D detector, $\sim 10 \mathrm{~Hz}$ data collection rate is expected, and our indexing algorithm will secure the simultaneous data analysis. In other words, the microstructural mapping of a typical $100 \mu \mathrm{m} \times 100 \mu \mathrm{m}$ area on a bulky sample surface can be completed in less than 20 min with $1 \mu \mathrm{m}$ spatial resolution. Secondly, combining a Si-drift detector and control software packages, polychromatic X-ray fluorescence signals will be collected in the meanwhile as micro-Laue diffraction scans are being carried out, so that the microstructural features can be correlated to the chemical compositions. Finally, in situ thermal, electrical, mechanical, gas environmental, and multi-field testing stages are under design and construction, and thus the materials' microstructural evolution under external stimuli can be investigated in real time.

\section{Received 9 January 2021; accepted 17 February 2021;} published online 14 May 2021

1 Friedrich W, Knipping P, Laue M. Interferenzerscheinungen bei Röntgenstrahlen. Ann Phys, 1913, 346: 971-988

2 Ice GE, Budai JD, Pang JWL. The race to X-ray microbeam and nanobeam science. Science, 2011, 334: 1234-1239

3 Larson BC, Yang W, Ice GE, et al. Three-dimensional X-ray structural microscopy with submicrometre resolution. Nature, 2002, 415: 887-890

4 Li R, Xie Q, Wang YD, et al. Unraveling submicron-scale mechanical heterogeneity by three-dimensional X-ray microdiffraction. Proc Natl Acad Sci USA, 2018, 115: 483-488

5 Chen K, Huang R, Li Y, et al. Rafting-enabled recovery avoids recrystallization in 3D-printing-repaired single-crystal superalloys. Adv Mater, 2020, 32: 1907164

6 Ma EY, Cui YT, Ueda K, et al. Mobile metallic domain walls in an all-in-all-out magnetic insulator. Science, 2015, 350: 538-541 
7 Cao J, Gu Y, Fan W, et al. Extended mapping and exploration of the vanadium dioxide stress-temperature phase diagram. Nano Lett, 2010, 10: 2667-2673

8 Tselev A, Budai JD, Strelcov E, et al. Electromechanical actuation and current-induced metastable states in suspended single-crystalline $\mathrm{VO}_{2}$ nanoplatelets. Nano Lett, 2011, 11: 3065-3073

9 Budai JD, Tselev A, Tischler JZ, et al. In situ X-ray microdiffraction studies inside individual $\mathrm{VO}_{2}$ microcrystals. Acta Mater, 2013, 61: 2751-2762

10 Chen K, Kunz M, Tamura N, et al. Residual stress preserved in quartz from the San Andreas Fault Observatory at Depth. Geology, 2015, 43: 219-222

11 Marcus MA, Amini S, Stifler CA, et al. Parrotfish teeth: Stiff biominerals whose microstructure makes them tough and abrasionresistant to bite stony corals. ACS Nano, 2017, 11: 11856-11865

12 Chen $\mathrm{K}$, Tamura N, Tang W, et al. High precision thermal stress study on flip chips by synchrotron polychromatic X-ray microdiffraction. J Appl Phys, 2010, 107: 063502

13 Lupinacci A, Chen K, Li Y, et al. Characterization of ion beam irradiated 304 stainless steel utilizing nanoindentation and Laue microdiffraction. J Nucl Mater, 2015, 458: 70-76

14 Zhou G, Pantleon W, Xu R, et al. Quantification of local dislocation density using 3D synchrotron monochromatic X-ray microdiffraction. Mater Res Lett, 2021, 9: 182-188

15 Ice GE, Larson BC. 3D X-ray crystal microscope. Adv Eng Mater, 2000, 2: 643-646

16 Kunz M, Tamura N, Chen K, et al. A dedicated superbend X-ray microdiffraction beamline for materials, geo-, and environmental sciences at the advanced light source. Rev Sci Instrum, 2009, 80: 035108

17 Chen X, Dejoie C, Jiang T, et al. Quantitative microstructural imaging by scanning Laue X-ray micro- and nanodiffraction. MRS Bull, 2016, 41: 445-453

18 Hocine S, Van Swygenhoven H, Van Petegem S, et al. Operando Xray diffraction during laser $3 \mathrm{D}$ printing. Mater Today, 2020, 34: $30-40$

19 Hofmann F, Song X, Dolbnya I, et al. Probing intra-granular deformation by micro-beam Laue diffraction. Procedia Eng, 2009, 1: 193-196

20 Hamilton JG, Reid JW, Feng R, et al. Evaluating synchrotron-based scanning Laue microdiffraction for mineralogy mapping in heterogeneous samples. ACS Earth Space Chem, 2018, 2: 1161-1167

21 Ulrich O, Biquard X, Bleuet P, et al. A new white beam X-ray microdiffraction setup on the BM32 beamline at the European Synchrotron Radiation Facility. Rev Sci Instrum, 2011, 82: 033908

22 Jiang MH, Yang X, Xu HJ, et al. Shanghai Synchrotron Radiation Facility. Chin Sci Bull, 2009, 54: 4171-4181

23 Li Y, Chen K, Dang X, et al. XtalCAMP: A comprehensive program for the analysis and visualization of scanning Laue X-ray micro-/ nanodiffraction data. J Appl Crystlogr, 2020, 53: 1392-1403

24 Brückner S. Estimation of the background in powder diffraction patterns through a robust smoothing procedure. J Appl Crystlogr, 2000, 33: 977-979

25 Chung JS, Ice GE. Automated indexing for texture and strain measurement with broad-bandpass X-ray microbeams. J Appl Phys, 1999, 86: 5249-5255

26 Pavese A. About the relations between finite strain in non-cubic crystals and the related phenomenological P-V equation of state. Phys Chem Miner, 2005, 32: 269-276

27 Kou J, Chen K, Tamura N. A peak position comparison method for high-speed quantitative Laue microdiffraction data processing. Scripta Mater, 2018, 143: 49-53

28 Li Y, Chen K, Tamura N. Mechanism of heat affected zone cracking in Ni-based superalloy DZ125L fabricated by laser 3D printing technique. Mater Des, 2018, 150: 171-181

29 Busing WR, Levy HA. Angle calculations for 3- and 4-circle X-ray and neutron diffractometers. Acta Cryst, 1967, 22: 457-464

30 Jiang L, Ye XX, Wang DJ, et al. Synchrotron radiation-based materials characterization techniques shed light on molten salt reactor alloys. Nucl Sci Tech, 2020, 31: 6

31 Wang W, Li C, Jiang L, et al. Evolution of carbide precipitates in Hastelloy $\mathrm{N}$ joints during welding and post weld heat treatment. Mater Charact, 2018, 135: 311-316

32 Zhou G, Zhu W, Shen H, et al. Real-time microstructure imaging by Laue microdiffraction: A sample application in laser 3D printed Ni-based superalloys. Sci Rep, 2016, 6: 28144

33 Li Y, Qian D, Xue J, et al. A synchrotron study of defect and strain inhomogeneity in laser-assisted three-dimensionally-printed $\mathrm{Ni}$ based superalloy. Appl Phys Lett, 2015, 107: 181902

34 Chen K, Kunz M, Li Y, et al. Compressional residual stress in Bastogne boudins revealed by synchrotron X-ray microdiffraction. Geophys Res Lett, 2016, 43: 6178-6185

35 Gäumann M, Bezençon C, Canalis P, et al. Single-crystal laser deposition of superalloys: Processing-microstructure maps. Acta Mater, 2001, 49: 1051-1062

36 Pham MS, Dovgyy B, Hooper PA, et al. The role of side-branching in microstructure development in laser powder-bed fusion. Nat Commun, 2020, 11: 749

Acknowledgements This work was supported by the National Key Research and Development Program of China (2016YFB0700404), and the National Natural Science Foundation of China (91860109, U2032205, 51671154, and 51927801). Chen K appreciates the support from the International Joint Laboratory for Micro/Nano Manufacturing and Measurement Technologies and the Collaborative Innovation Center of High-End Manufacturing Equipment.

Author contributions Tai R and Huang $\mathrm{X}$ proposed and supervised this work. Liu M, Dong X and Li ZL designed and built the micro-Laue beamline on BL09B. Jiang L, Yan S, Li L and Li ZJ prepared the superalloy specimens and conducted the $2 \mathrm{D}$ scans. Ren C, Kou J and Chen $\mathrm{K}$ developed the software packages, analyzed the data and interpreted the results. Chen K, Ren C, Li ZJ, Li ZL and Huang X wrote the paper. All authors contributed to the discussion.

Conflict of interest The authors declare that they have no conflict of interest.

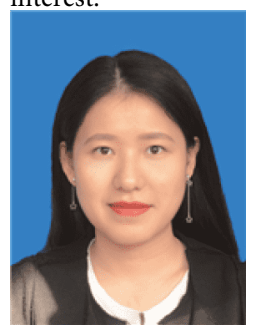

Chenyu Ren is currently a $\mathrm{PhD}$ student at the Center for Advancing Materials Performance from the Nanoscale (CAMP-Nano), Xi'an Jiaotong University. Her research interests focus on the microstructure study of laser-processed $\mathrm{Ni}$ based superalloys. 


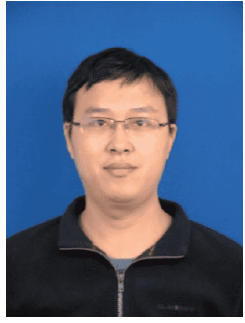

Li Jiang obtained his Master degree from Northwestern Polytechnical University in 2012 and $\mathrm{PhD}$ degree from the University of Chinese Academy of Sciences in 2017. He is now an associate professor at Shanghai Institute of Applied Physics, Chinese Academy of Sciences. His research interests focus on the design of superalloys for nuclear applications and the alloy corrosion behavior by fission products using advanced material characterization approaches such as synchrotron radiation-based techniques.

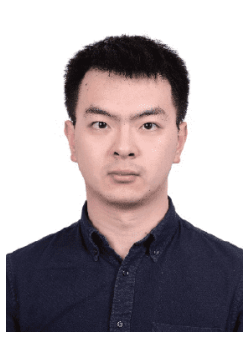

Jiawei Kou obtained his Bachelor degree in 2018 and then continued his graduate study at the CAMP-Nano, Xi'an Jiaotong University. He is dedicated to the development of high-speed indepth Laue diffraction data mining algorithms and software packages. He applies these new approaches to the study of the microstructures of advanced alloys.

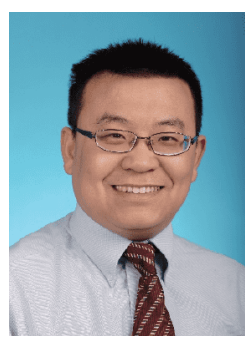

Kai Chen obtained his Bachelor and $\mathrm{PhD}$ degrees from Peking University and the University of California Los Angeles in 2005 and 2009, respectively. After two postdoctoral stints at the University of California Berkeley and Lawrence Berkeley National Laboratory, he has been a professor at Xi'an Jiaotong University since 2011. $\mathrm{He}$ is interested in pushing the software and hardware developments for synchrotron microLaue diffraction and applying this advanced characterization method to the study of advanced engineering materials.

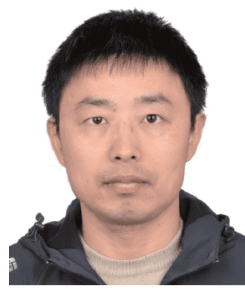

Zhongliang Li obtained his $\mathrm{PhD}$ degree from the National Synchrotron Radiation Laboratory, University of Science and Technology of China in 2011. He is currently an associate professor in the optical group of Shanghai Synchrotron Radiation Facility. His research interests focus on the X-ray measurement methodology of beamline instruments and optical elements, synchrotron radiation-based science and technology, and the microstructural evolution of alloys under corrosive conditions of fission products.

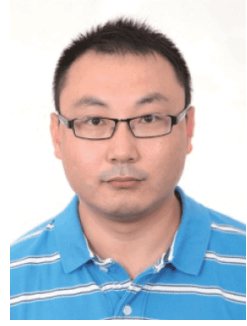

Zhijun Li obtained his PhD degree from Harbin Institute of Technology in 2007. He holds the professorship at Shanghai Institute of Applied Physics, Chinese Academy of Sciences. His research interests focus on the design and characterization of alloys for nuclear energy applications using advanced synchrotron radiation-based techniques.

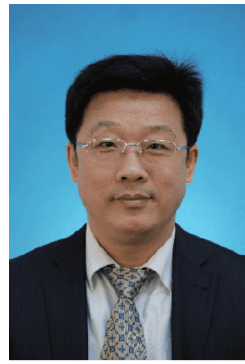

Renzhong Tai obtained his PhD degree from the Graduate University for Advanced Studies, Japan in 1999. After postdoctoral research on coherent X-ray applications at Japan Atomic Energy Research Institute, he holds the professorship at Shanghai Institute of Applied Physics, Chinese Academy of Sciences. He is currently the executive deputy director of Shanghai Synchrotron Radiation Facility. His research interests focus on synchrotron radiation science and techniques, $\mathrm{X}$ ray microscopy, X-ray detector technology, X-ray interference lithography, photon correlation spectroscopy, and beamline development.

\section{上海光源微束劳厄技术的发展及其在材料科学研 究中的应用}

任晨宇 ${ }^{1 \dagger}$, 蒋力 ${ }^{2 \dagger}$, 寇嘉伟 ${ }^{1 \dagger}$, 间帅 ${ }^{3}$, 李丽 $^{3}$, 刘孟廷 $^{3}$, 董晓浩 ${ }^{3}$, 陈凯 ${ }^{1 *}$, 李中亮 ${ }^{3 *}$, 李志军 ${ }^{2^{*}}$, 黄晓旭 ${ }^{4}$, 郃仁忠 ${ }^{3^{*}}$

摘要 同步辐射微束劳厄技术以其超高的晶体取向和晶格应力/应 变分辨率而在材料科学研究中显示出重要的应用潜力. 本文报道 了我国上海光源在微束劳厄技术方面的最新进展. 目前已获得 40 $\times 50 \mu \mathrm{m}^{2}$ 的X射线束斑尺寸, 在我国(不含台湾省)首次实现了白光 微束劳厄二维扫描实验, 利用我国首套完全独立研发、具有自主 知识产权的数据分析软件系统, 成功定量研究了镍基高温合金 GH3535母材与焊缝区域晶体取向、应变和显微缺陷等微观组织 结构特征, 并提出了后续线站优化设计方案. 在未来工作中, 将进一 步提高空间分辨率，加入X射线荧光分析与原位测试装置. 上海光 源微束劳厄射线站将为材料科学研究提供更高的分辨率和更多的 测试表征功能与能力. 